\title{
Neurologic Disorder Masquerading as Postpregnancy Progression of Keratoconus
}

\author{
Rohit Shetty, Sharon D’Souza, Vardhaman P Kankariya, Samaresh Srivastava, Viraj Vasavada, Kareeshma Wadia
}

\begin{abstract}
A 28-year-old woman with bilateral stable keratoconus for 2 years came with a complaint of recent blurring of vision 4 weeks postpartum. Her best-corrected visual acuity had dropped by three lines in the right eye and two lines in the left eye with the same manifest refraction. Corneal topography and Scheimpflug imaging revealed mild progression of the keratoconus, but not enough to explain the drop in visual acuity. However, fundus examination revealed temporal disk pallor in both eyes. Visual field analysis revealed classic bitemporal hemianopia. Subsequently, magnetic resonance imaging (MRI) of brain was performed which revealed a pituitary macroadenoma with pituitary apoplexy that was impinging on the pons. Based on these findings, urgent neurosurgery was performed, and the patient regained her visual acuity and fields 2 weeks later. Neuro-ophthalmologic disorders should always be kept as a differential diagnosis in pregnantand postpartum women. A high index of suspicion from the ophthalmologist can often avert life-threatening problems.
\end{abstract}

Keywords: Pituitary apoplexy, Keratoconus, Masquerade.

Key message: Neuro-ophthalmologic disorders can masquerade as a variety of clinical presentations in pregnant and postpartum women. A high index of suspicion from the ophthalmologist can often avert life-threatening problems.

How to cite this article: Shetty R, D'Souza S, Kankariya VP, Srivastava S, Vasavada V, Wadia K. Neurologic Disorder Masquerading as Postpregnancy Progression of Keratoconus. Int J Kerat Ect Cor Dis 2012;1(3):205-208.

Source of support: Nil

Conflict of interest: None declared

\section{INTRODUCTION}

Pregnancy is often associated with ocular changes which may be more commonly transient but occasionally, permanent. The ocular effects of pregnancy may be physiological or pathological or may be modifications of pre-existing conditions. ${ }^{1}$ Physiological changes include changes in the cornea and refractive status. Pre-existing conditions, such as keratoconus may progress during pregnancy. ${ }^{2,3} \mathrm{M}$ oreover, neuro-ophthalmological disorders should be kept in mind in pregnant women presenting with visual acuity or field loss.

We report the case of a 28-year-old female with keratoconus who presented in the early postpartum period with what seemed like progression of keratoconus, but was detected to have pituitary adenoma on detailed investigations.

\section{CASE REPORT}

A 28-year-old female, known to have keratoconus for the last 2.5 years, presented to us with complaints of worsening of vision, and difficulty viewing her mobile phone, progressively worsening since 4 months. She was 4 weeks postpartum at this time. Her pregnancy had been uneventful, with no history of ocular complains, health problems and had a full-term normal delivery.

She had presented to us for the first time 2.5 years back. Her uncorrected distance visual acuity (UDV A) was 20/200 in the right eye and 20/30 in the left eye, that corrected to 20/ 20 in the right eye (+1.5 D Sph/-5.0 D Cyl@ 60) and 20/15 in the left eye (+0.5 D Sph/-1.0 D Cyl@90). Placido disk and scanning slit-based videokeratography (Orbscan, B ausch and L omb), rotating Scheimpflug imaging (Pentacam, Oculus, Germany), slit-lamp biomicroscopy and fundoscopy was performed in both eyes. She was diagnosed to have diagnosed to have keratoconus in the right eye (Stage II, A msler$\mathrm{K}$ rumeich classification) and forme fruste keratoconus in the left eye (Figs $1 A$ to $D$ ). She was given contact lenses and followed up. At 1-year follow-up, corrected distance visual acuity (CDVA) in the right eye was 20/20 $(+1.5 \mathrm{D}$ Sph/-5.0 D Cyl@ 60) and in the left eye was 20/20 (+1.0 D Sph/-2.0 D Cyl@100) with minimal progression. She remained stable subsequently for the next year as well.

Currently, her visual acuity had dropped to 20/63 $(+2.0 \mathrm{D}$ Sph/-5.5 D Cyl@60) in the right eye and 20/32 (+1.5 D Sph/-2.5 D Cyl@100) in the left eye, with just a minor change in the refraction. Topography and Scheimpflug imaging showed minimal progression in both eyes, with the predominant change seen on the posterior el evation float (Figs $1 A$ to $D$ ).

However, her specific complaint of difficulty in reading her mobile phone could not be correlated with this degree of progression al one. Fundus evaluation reveal ed temporal disk pallor in both eyes. Humphrey visual field analysis revealed bitemporal, congruous hemianopia (Fig. 2). She was immediately referred to a neurol ogist and magnetic resonance imaging (M RI) of brain revealed a nonfunctioning pituitary macroadenoma with hemorrhage and fluid levels (Fig. 3).

She was advised and underwent urgent surgical transsphenoidal decompression on the same day. Postoperatively, at 3 weeks, her vision improved to 20/30 in both eyes and visual field analysis showed partial resolution of the field defects (Fig. 4).

\section{DISCUSSION}

$V$ isual acuity loss during or after pregnancy needs a high index of suspicion. In this case, the first differential 

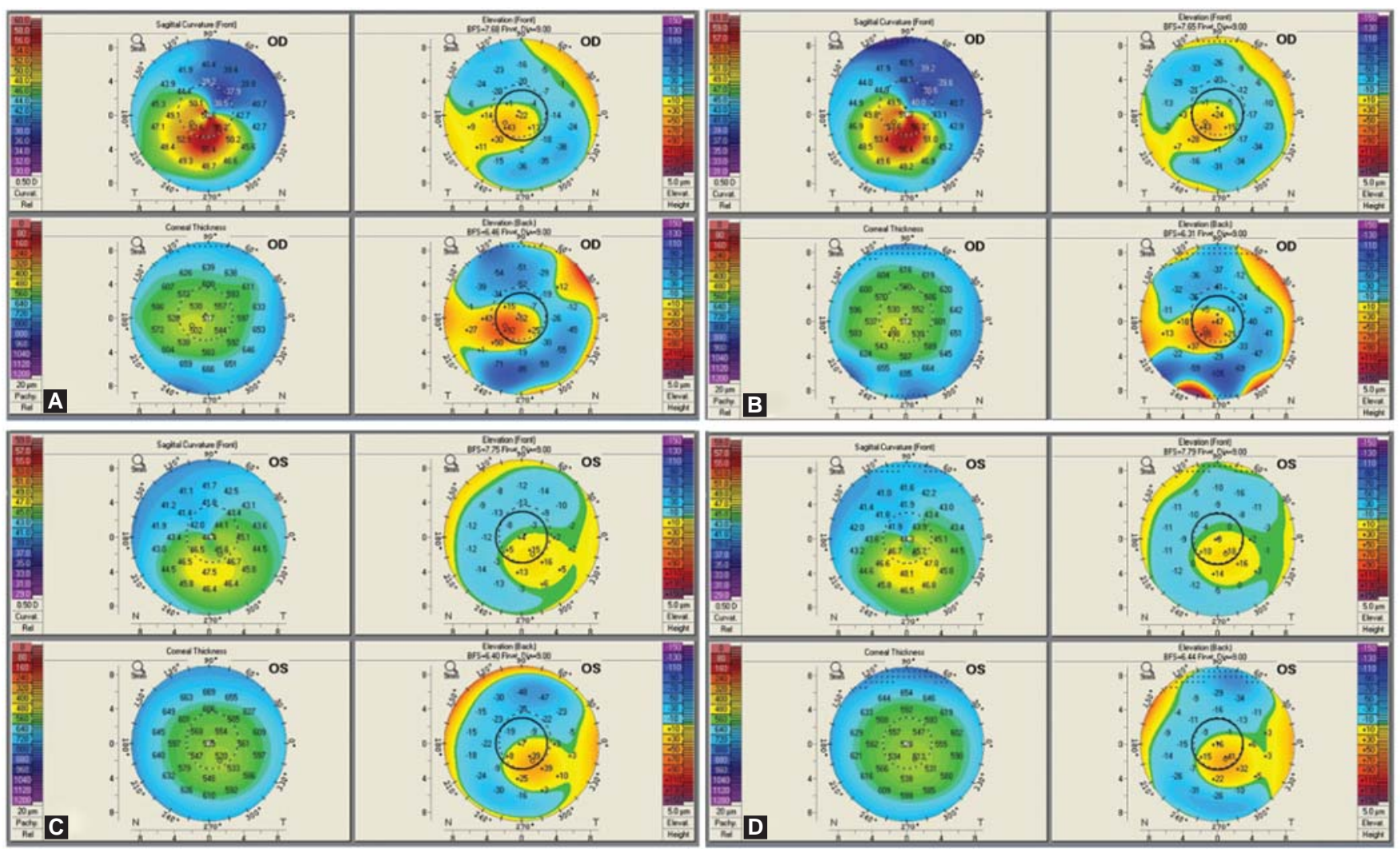

Figs 1A to D: Scheimpflug imaging of right eye at baseline $(A)$ and presentation $(B)$ left eye at baseline $(C)$ and presentation (D) showing some progression of the keratoconus in both eyes
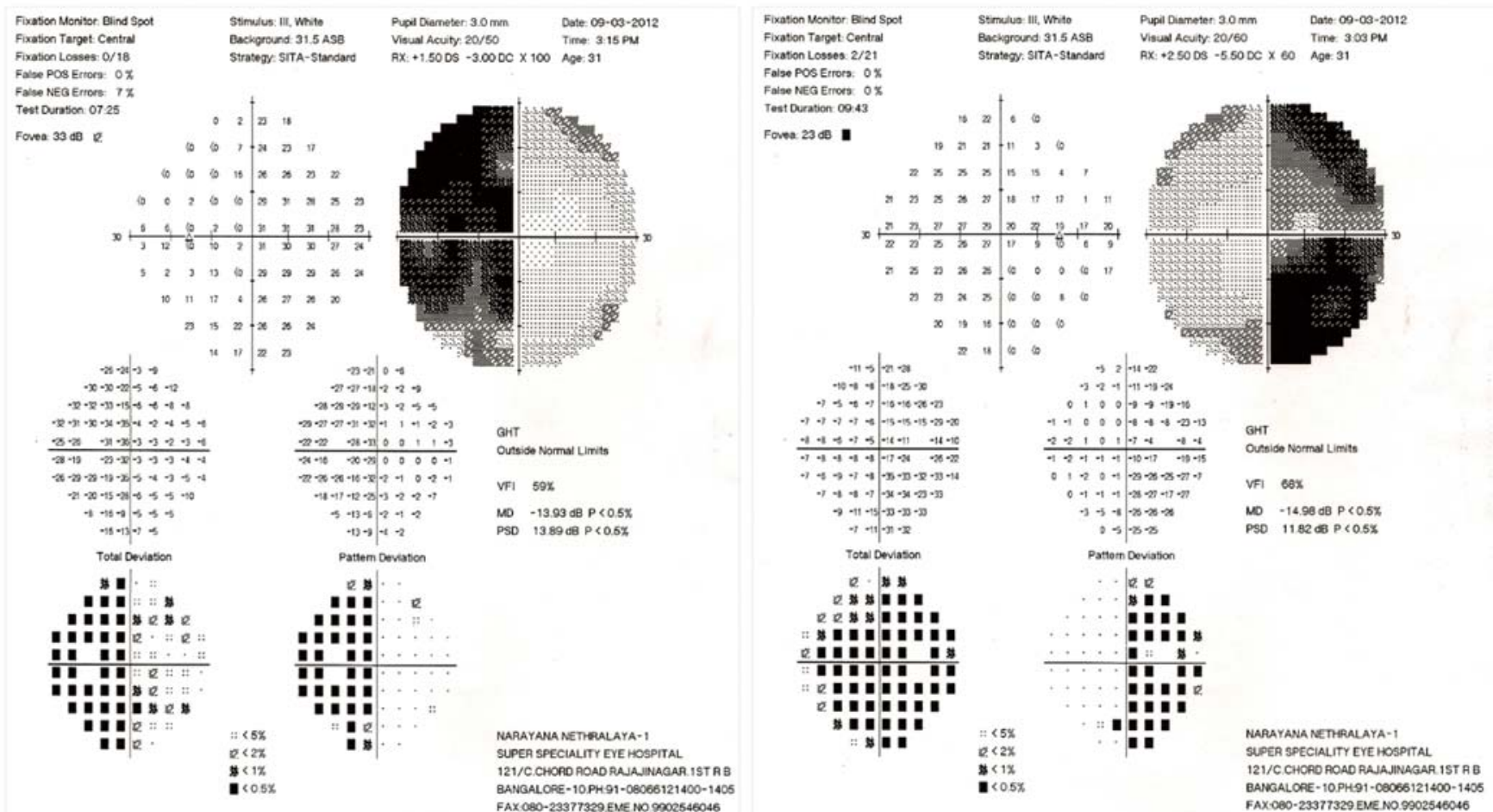

Fig. 2: Humphrey visual field analysis revealing classic bitemporal hemianopia

diagnosis to come to mind would be a progression of the keratoconus. Hormonal changes during pregnancy may affect corneal biomechanics negatively, and pregnancy may be an underestimated risk factor for progression of keratoconus. ${ }^{2}$ In a previous case series, ${ }^{2}$ the authors showed pregnancy-induced keratoconus progression in patients with no accompanying disease. Changes in estrogen levels may play a role in corneal ectasia. A significant stiffness-reducing 

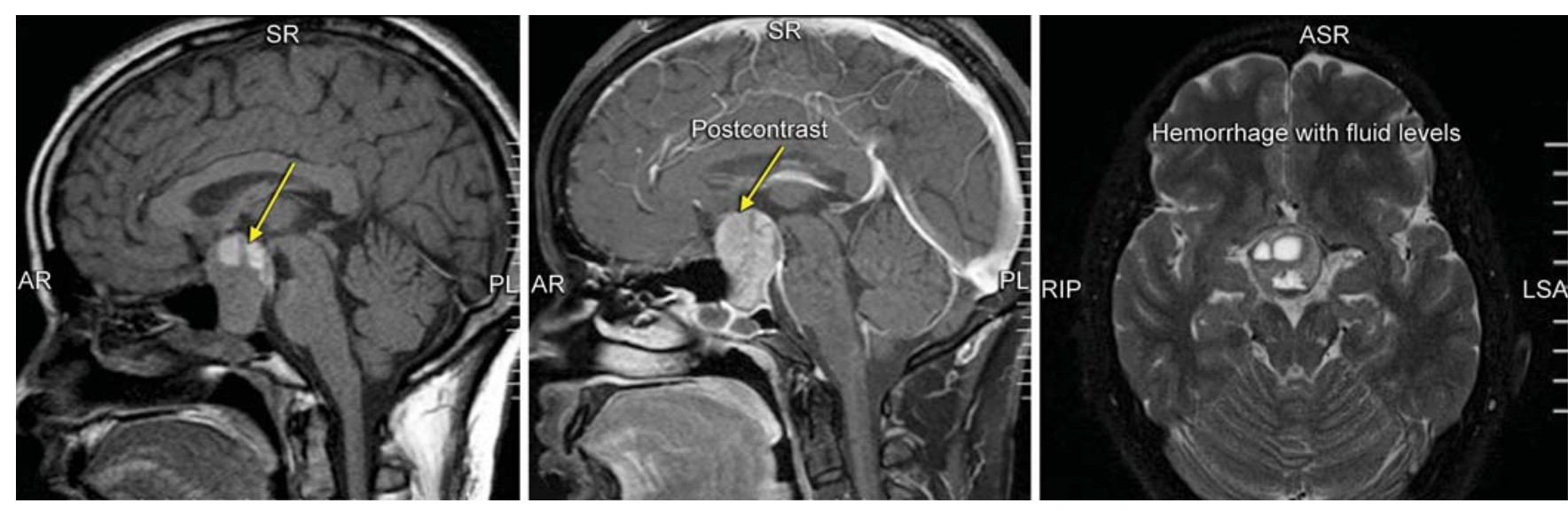

Fig. 3: MRI brain showing pituitary adenoma with fluid level, suggestive of hemorrhage
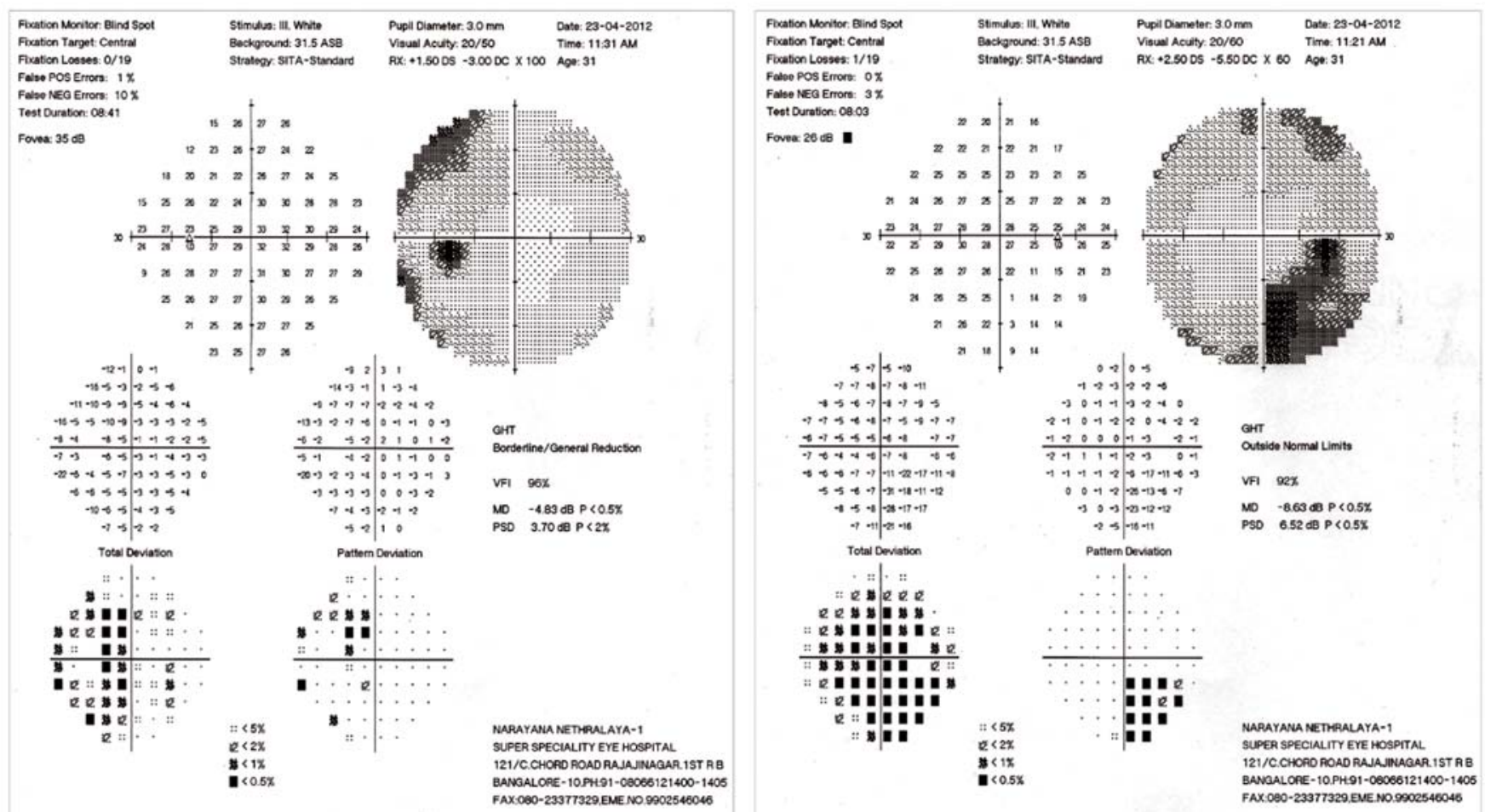

Fig. 4: Resolution of visual field defects following resection of the adenoma

effect of estrogen on cornea has been shown, which suggests that high estrogen states, such as pregnancy may predispose biomechanically weak corneas to ectasia development or progression. ${ }^{4,5}$ In addition, various studies have demonstrated that serum levels of matrix metalloproteinases (M M Ps) are increased and serum levels of tissue inhibitors of MMPs (TIMPs) are decreased during pregnancy. ${ }^{6} \mathrm{~A}$ case of hypothyroxinemia-induced acute corneal hydrops during pregnancy, ${ }^{7}$ has also been reported. The authors suggested that the progression was related to thyroid gland dysfunction.

In this case, CDVA had significantly deteriorated despite a stable refraction. M oreover, the complaint of visual loss in a particular direction in a patient with longstanding compensated keratoconus could not be explained by mild progression of the cone. A careful fundus evaluation and visual field analysis hel ped us clinch the diagnosis at a very crucial point.

This was an atypical presentation of pituitary apoplexy masquerading as a progressive keratoconus. Neuroophthalmological disorders, such as venous sinus thrombosis, benign intracranial hypertension, pituitary adenoma, meningioma and optic neuritis should be kept in mind as differential diagnosis in pregnant and early postpartum women. ${ }^{1}$ Physiologic changes in pregnancy make vascular conditions more frequent, including retinal artery occlusion and pituitary apoplexy. Therefore, women presenting with visual acuity loss, visual field loss, persistent headaches or oculomotor palsies should be examined for the above possibilities. 
Pituitary apoplexy is a potentially life-threatening emergency. Pituitary apoplexy occurs when an existing pituitary adenoma undergoes acute hemorrhage, infarct or both. The reported incidence of pituitary tumor apoplexy is usually less than $5 \%$ with a range between 0.6 and $10 \%$. Common predisposing factors include closed head trauma, hypotension, hypertension, history of pituitary irradiation, cardiac surgery, anticoagulant therapy and pregnancy. ${ }^{8,9}$ Clinical features of pituitary apoplexy include sudden onset of headache, nausea, vomiting, visual symptoms, ptosis, altered mental status and endocrinologic dysfunction. The tumor and intrasellar contents may expand superiorly, compressing the optic chiasm, optic tracts, and optic nerves, producing decreased visual acuity in $52 \%$ and visual field defects in $64 \%$ of patients.

Although rare, visual symptoms could be the early presenting features, as in the case here. If the ophthal mologist does not maintain a high index of suspicion, timely diagnosis of such cases can be missed. Sellar M RI is a sensitive study for imaging the pituitary gland. ${ }^{10}$ $M$ anagement includes emergently stabilizing patient's symptoms. M any patients will require intravenous fluids and blood transfusions. It is crucial that high dose corticosteroids are also administered to replace endogenous hormone deficiency and to prevent edema on parasellar structures. Often, urgent trans-sphenoidal surgical decompression is required as definitive treatment. ${ }^{10}$ Improvement in visual field, visual acuity and diplopia is typically observed after therapy. Preoperative preservation of at least light perception vision predicts better visual outcomes postoperatively compared with patients with absence of light perception. Surgery within the first week is associated with better visual outcome when compared with delayed surgery.

\section{CONCLUSION}

Neuro-ophthalmic conditions can masquerade themselves into varied presentations. Therefore in pregnant and postpartum women, a high index of suspicion and a thorough ophthalmic evaluation can of ten be vision and life saving.

\section{REFERENCES}

1. Garg P, A ggarwal P. O cular changes in pregnancy. Nepal J Ophthalmol 2012;4:150-61.

2. Bilgihan K, Hondur A, Sul S, Ozturk S. Pregnancy-induced progression of keratoconus. Cornea 2011;30:991-94.
3. Padmanabhan P, Radhakrishnan A, Natarajan R. Pregnancytriggered iatrogenic (post-laser in situ keratomileusis) corneal ectasia: A case report. Cornea 2010;29:569-72.

4. Suzuki T, K inoshita Y, Tachibana M, M atsushima $Y, K$ obayashi $Y, A$ dachi $W$, et al. Expression of sex steroid hormone receptors in human cornea. Curr Eye Res 2001;22:28-33.

5. Spoerl E, Zubaty V, Raiskup-W olf F, Pillunat LE. O estrogeninduced changes in biomechanics in the cornea as a possible reason for keratectasia. Br J O phthalmol 2007;91:1547-50.

6. Clark IM, M orrison J J, Hackett GA, Powell EK, Cawston TE, Smith SK. Tissue inhibitor of metalloproteinases: Serum levels during pregnancy and labor, term and preterm. O bstet Gynecol 1994;83:532-37.

7. Gatzioufas Z, Thanos S. A cute keratoconus induced by hypothyroxinemia during pregnancy. J Endocrinol Invest 2008;31:262-66.

8. Nawa R, A bdel M annan D, Selman W, A rafah B. Pituitary tumor apoplexy: A review. J Intens Care M ed 2008;23:75-90.

9. Kim J, Park B, K im S, Lim Y . Pituitary apoplexy due to pituitary adenoma infarction. J Korean Neurosurg Soc 2008;43:246-49.

10. Murad-Kejbou S, Eggenberger E. Pituitary apoplexy: Evaluation, management and prognosis. Curr Opinion in Ophthalmol 2009;20:456-61.

\section{ABOUT THE AUTHORS}

\section{Rohit Shetty (Corresponding Author)}

Head, Department of R efractive Surgery and Neuro-ophthalmology Narayana Nethralaya Super Speciality Hospital and Postgraduate Institute, B engaluru, K arnataka, India, e-mail: drrohitshetty@yahoo.com

\section{Sharon D'Souza}

Consultant, D epartment of Cornea and R efractive Surgery, N arayana Nethralaya Superspeciality Hospital and Postgraduate Institute Bengaluru, K arnataka, India

\section{Vardhaman P Kankariya}

Director, Department of Cornea and R efractive Surgery, Sai Surya Netra Seva, A hmednagar, M aharashtra, India

\section{Samaresh Srivastava}

Consultant, Department of Cataract and IOL, Raghudeep Eye Clinic A hmedabad, Gujarat, India

\section{Viraj Vasavada}

Consul tant, D epartment of Cataract and IOL , R aghudeep Eye H ospital A hmedabad, Gujarat, India

\section{Kareeshma Wadia}

Fellow, Department of Cornea and Refractive Surgery, Narayana Nethralaya Super Speciality Hospital and Postgraduate Institute Bengaluru, Karnataka, India 